\title{
ERRATUM
}

\section{Volume 77, no. 17, 2003, Table of Contents and Author Index}

Table of Contents and Author Index: "Stéphanie Villet" is the correct spelling for the list of authors for the article "Maedi-Visna Virus and Caprine Arthritis Encephalitis Virus Genomes Encode a Vpr-Like but No Tat Protein,” p. 9632-9638. 